Conclusions DUD is prevalent in this population and associated with personal, social, and community exposures. Among those with DUD, diagnosis with multiple drug use disorders was common; network analyses showed several large partial correlations between substances, with cocaine and prescription opioid use disorder co-diagnosis being the most strongly associated.

Significance DUD is linked to a variety of injuries including suicide, partner violence, firearm violence, death by homicide, and violence/injury in general. Understanding the relationship between co-occurring DUDs and associated risk factors allow for targeted intervention strategies.

\section{TBI/Concussion injuries}

\section{OBJECTIVE MEASURES OF VISUAL AND AUTONOMIC SYSTEM DEFICITS FOLLOWING CONCUSSION IN ADOLESCENTS}

\begin{abstract}
${ }^{1,2} \mathrm{D}$ Jain, ${ }^{2,3} \mathrm{CC}$ MCDonald, ${ }^{2} \mathrm{OE}$ Podolak, ${ }^{2} \mathrm{~F}$ Mohammed, ${ }^{2,4,5} \mathrm{CL}$ Master, ${ }^{2,4} \mathrm{~KB}$ Arbogast. ${ }^{1}$ University of Pennsylvania, Philadelphia, USA; ${ }^{2}$ Center for Injury Research and Prevention, the Children's Hospital of Philadelphia, Philadelphia, USA; ${ }^{3}$ University of Pennsylvania, School of Nursing, Philadelphia, USA; ${ }^{4}$ Perelman School of Medicine, University of Pennsylvania, Philadelphia, USA; ${ }^{5}$ Sports Medicine and Performance Center, Children's Hospital of Philadelphia, Philadelphia, USA
\end{abstract}

\subsection{6/injuryprev-2021-SAVIR.32}

Statement of purpose Visual and autonomic system disturbances are common sequelae of concussion. Clinical concussion diagnosis currently relies on subjective assessments such as symptom questions such as the Post-Concussion Symptom Inventory (PCSI). Quantification of visual and autonomic dysfunction could provide an objective method of acute diagnosis and subacute identification of ongoing injury.

Methods/Approach We collected objective eye tracking data from three cohorts ages 12-17 years: healthy controls $(n=105$, mean age: 15.3, 56.2\% female), concussed cases seen within 28 days of injury (acute, $\mathrm{n}=125$, mean days since injury:12.5, mean age: $15.4,46.4 \%$ female) and concussed cases seen within 2990 days of injury (sub-acute, $\mathrm{n}=94$, mean days since injury: 53.6, mean age: 15.4, 70.2\% female). We compared selfreported symptoms and eye tracking metrics across groups using a series of chi-square analyses and one-way analysis of variance, with Tukey's range test for post-hoc testing. Bonferroni corrections were used to account for multiple comparisons.

Results Controls reported significantly lower PCSI scores (mean \pm SD: $6.0 \pm 9.1)$ than acute cases $(31.2 \pm 24.7, \mathrm{p}<$ $0.001)$ and sub-acute cases $(31.4 \pm 27.8, \mathrm{p}<0.001)$. A significantly lower proportion of controls experienced symptoms after completing the assessment (10\%) than acute cases $(55 \%$, $\mathrm{p}<0.001)$ and sub-acute cases (38\%, p < 0.001). Six eye tracking metrics related to pupil diameter (left and right pupil size mean and median values, mean and median differences in left and right pupil size), were significantly greater among acute and sub-acute cases compared to controls.

Conclusion Measures of pupil diameter were greater for acute and sub-acute cases compared to healthy controls, suggesting autonomic dysfunction post-injury. No other metrics related to eye movement were discriminatory in this cohort, likely due to the heterogeneity of concussion.

Significance These findings support the quantification of visual and autonomic dysfunction as objective markers of pediatric concussion.

\section{Alcohol and substance abuse}

\section{AN ANALYSIS OF DRUG OVERDOSE DEATHS BY INTENT IN OHIO FROM 2016-2018}

D Saunders, A Hagemeyer, L Werhan. Ohio Department of Health, Columbus, USA

\subsection{6/injuryprev-2021-SAVIR.33}

Statement of purpose In 2018, unintentional drug overdose was the leading cause of injury-related deaths among Ohioans. In the same year, drug poisoning was the third leading mechanism of suicide deaths. This session will review the 'who, when, where, and how' of overdose deaths from the Ohio Violent Death Reporting System (OH-VDRS) to provide participants with an understanding of the sociodemographic characteristics and related circumstances (e.g., prior suicide attempts, prior mental health or substance use disorder treatment, co-occurring mental health conditions) surrounding overdose deaths.

Methods/Approach Descriptive statistics of 2016-2018 OHVDRS data assessed sociodemographic characteristics of and circumstances preceding deaths by unintentional and intentional (i.e., suicide) drug overdose among Ohio residents who died in Ohio.

Results Regardless of intent, most drug overdose decedents were Caucasian (unintentional: $84.6 \%$ versus intentional: 95.3\%) with a high school degree or less (unintentional: $77.4 \%$ versus intentional: $62.5 \%$ ). While unintentional drug overdose decedents were more likely to be male (67.0\%) and 25-34 years old (27.8\%), most intentional drug overdose decedents were female $(54.9 \%)$ and $45-54$ years old (26.3\%). Regarding circumstances, a larger proportion of unintentional drug overdose decedents had a substance abuse problem $(86.2 \%$ versus $27.3 \%$ ), while a larger proportion of intentional drug overdose decedents had a mental health condition (78.9\% versus $43.3 \%)$. Toxicology differences were noted; antidepressants, anticonvulsants, antipsychotics, and benzodiazepines were identified as a cause of death in a higher proportion of intentional drug overdose deaths, while cocaine and opioids were identified in more unintentional drug overdose deaths.

Conclusion Examining drug overdose trajectories by intent can better inform interventions by targeting diverse prevention strategies to the appropriate populations.

Significance A better understanding of drug overdose trajectories by intent could provide evidence to guide the data-driven decision making surrounding the development and implementation of evidence-based policies, programs, and interventions.

\section{Homicide/Assault}

\section{SOCIAL AND PHYSICAL ENVIRONMENTAL DETERMINANTS OF VIOLENT HOMICIDE DEATH INCIDENCE: A SPATIAL ECOLOGICAL CASE-CONTROL STUDY OF US ZIP CODES}

A Gobaud, C Mehranbod, C Morrison. Columbia University Mailman School of Public Health, New York, USA

\subsection{6/injuryprev-2021-SAVIR.34}

Statement of purpose Violent homicide is a major cause of death and a driver of health disparities in the US. It is not 